Recherches en histoire de l'art, histoire des civilisations, archéologie, anthropologie et muséologie

$6 \mid 2015$

Cahiers 6

\title{
La collection extrême-orientale du musée Adrien Dubouché de Limoges
}

The Far East collection of the Musée Adrien Dubouché de Limoges

\section{Pauline d'Abrigeon}

\section{(2) OpenEdition}

Journals

Édition électronique

URL : http://journals.openedition.org/cel/317

DOI : $10.4000 / \mathrm{cel} .317$

ISSN : 2262-208X

Éditeur

École du Louvre

Référence électronique

Pauline d'Abrigeon, «La collection extrême-orientale du musée Adrien Dubouché de Limoges », Les Cahiers de l'École du Louvre [En ligne], 6 | 2015, mis en ligne le 01 avril 2015, consulté le 17 septembre 2019. URL : http://journals.openedition.org/cel/317 ; DOI : 10.4000/cel.317

\section{c) (†) $\odot$}

Les Cahiers de l'École du Louvre sont mis à disposition selon les termes de la licence Creative Commons Attribution - Pas d'Utilisation Commerciale - Pas de Modification 4.0 International. 


\section{La collection extrême-orientale du musée Adrien Dubouché de Limoges}

\section{Pauline d'Abrigeon}

Les céramiques extrême-orientales, et plus particulièrement les porcelaines chinoises, ont fait l'objet d'une véritable fascination en Europe. Importées par les Compagnies des Indes Orientales (entre autres néerlandaises telle que la $\mathrm{VOC}^{1}$ ), elles prennent place dans les salons les plus en vue du XviII ${ }^{\mathrm{e}}$ siècle, initiant la mode des chinoiseries ${ }^{2}$. À l'aube du XIX ${ }^{e}$ siècle, la création des musées change radicalement la manière de percevoir, d'exposer, et d'appréhender ces objets. Le propos de cet article n'est pas de retracer l'histoire de la production de ces objets du point de vue de l'histoire de l'art, et selon les valeurs que cette discipline leur attribue, mais plutôt de "suivre les choses elles-mêmes ", en puisant dans le " fétichisme méthodologique ", défini par l'anthropologue Arjun Appadurai dans The Social Life of Things ${ }^{3}$. L'objet de cette étude est d'envisager, à travers l'histoire des collections, les différents statuts ${ }^{4}$ attribués à l'objet.

Par ailleurs, comprendre les changements dans la perception de l'objet nécessite de bien saisir le rôle indispensable qu'ont joué les musées. La mutation des procédés de monstration, ainsi que le développement du tissu de connaissances accompagnant la muséalisation ${ }^{5}$ de ces pièces ont un impact particulier sur la façon d'appréhender les œuvres extrême-orientales.

Cette étude se fonde sur l'exemple du musée Adrien Dubouché de Limoges qui, en plus de s'inscrire dans la mouvance des musées d'art industriel, possède une collection d'art extrême-oriental particulièrement riche et originale.

\section{Les musées d'art industriel, écrins originaux des céramiques extrême-orientales au XIX ${ }^{\mathrm{e}}$ siècle}

\section{Caractéristiques, origine et développement des musées d'art industriel}

Musée d'art industriel... l'appellation n'a que peu de résonance dans le sens commun, tant il est vrai que l'adjectif «industriel» a pris de nos jours une connotation péjorative associée à la production de masse. Ces établissements s'érigent pourtant dans la seconde moitié du XIX ${ }^{e}$ siècle comme les vitrines de toutes les prouesses techniques dans des domaines aussi variés que l'ingénierie ou les arts décoratifs. Ils ont pour caractéristiques de présenter des collections anciennes et de l'époque, et d'exposer le meilleur des industries qu'il s'agisse de porcelaine, de tapisserie, de machines, ou d'autres inventions. Ils sont pour ainsi dire, le produit " en dur " des Expositions universelles qui jalonnent le siècle, à ceci près que ces dernières mettaient en concurrence éphémère les nations quand les musées offraient des modèles permanents aux industries. Beaucoup de ces musées virent cependant le jour à la suite d'une Exposition universelle, le premier et modèle du genre restant

1. Cet article est le fruit d'une vacation scientifique financée par Musée national de la porcelaine Adrien Dubouché - Cité de la Céramique, I'auteur remercie vivement Chantale Meslin-Perrier, ainsi que Céline Paul, pour avoir permis et encouragé ce travail ; que soit également remercié François Mairesse, pour ses relectures.

Acronyme pour Vereenigde Oost-Indische, littéralement: «Compagnie unie des Indes Orientales ».

2. Voir à ce propos Dawn Jacobson, Chinoiserie, Londres, Phaidon Press, 1993, mais également, en langue française : cat. d'exp., Pagodes et dragons, Exotisme et fantaisie dans l'Europe rococo 1720-1770, sous la direction de Georges Brunel, musée Cernuschi, 24 février-17 juin 2007, Paris, Paris Musées, 2007.

3. Arjun Appadurai, The Social Life of Things: Commodities in Cultural Perspective, New York, Cambridge University Press, 1986, p. 5.

4. Sur le concept de transformation du sens lié à la céramique chinoise, voir Stacey Pierson, From Object to Concept, Global Consumption and the Transformation of Ming Porcelain, Hong Kong, Hong Kong University Press, 2013.

5. Sur la définition de la notion de muséalisation voir André Desvallées et François Mairesse (dir.), Dictionnaire encyclopédique de muséologie, Paris, Armand Colin, 2011, pp. 262, 263. 
le South Kensington Museum de Londres (actuel Victoria and Albert Museum) fondé après l'Exposition de 1851 (fig. 1). On peut également citer le Musée pour l'Art et l'Industrie de Vienne, le Musée des Arts Industriels de Berlin (1867), le Musée des Arts appliqués de Budapest (1872) et le musée du Trocadéro (1878) ${ }^{6}$.

Fig.1

Tallis's history and description of the Crystal Palace and the exhibition of the world's industry in 1851 Londres

(c) Heidelberg University Library, Tallis's history and description of the Crystal Palace and the exhibition of the world's industry in 1851

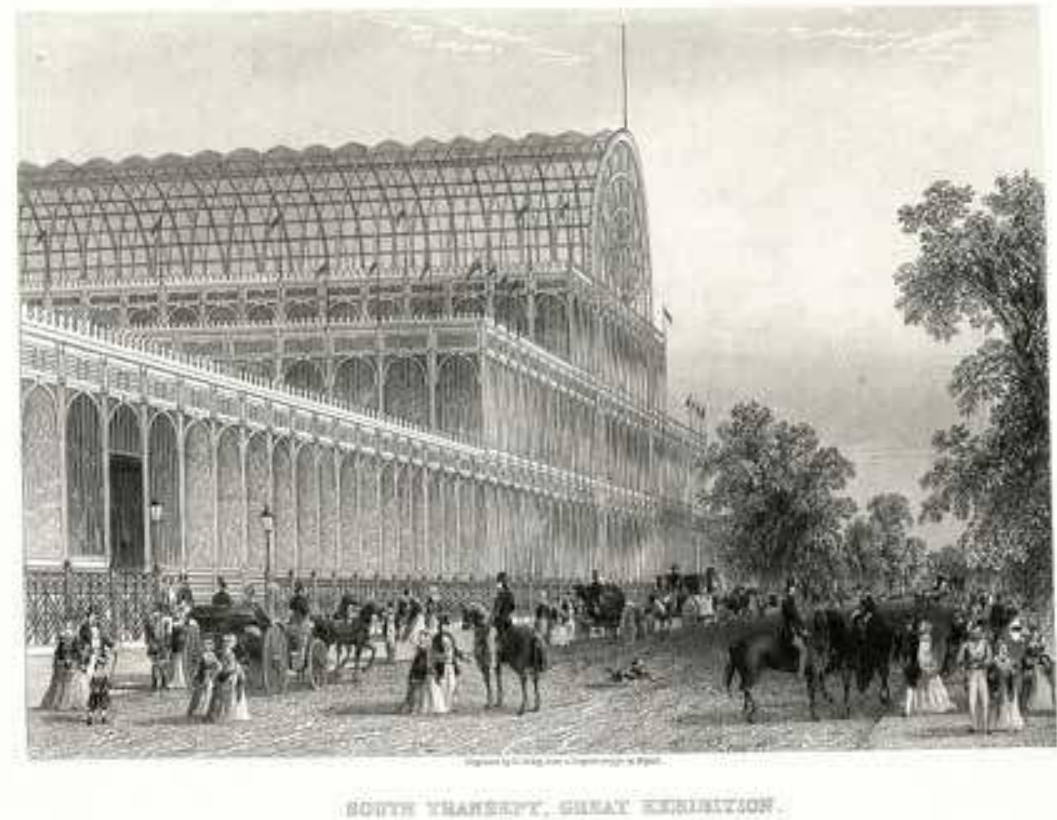

Ces musées ont également pour trait commun d'avoir de fortes visées éducatives : le musée de South Kensington demeure historiquement lié à une école de design, son fonds ayant pour origine la petite collection rassemblée par la School of Design, créée en 18367 . Il a toujours entretenu un lien étroit avec cette école et la création contemporaine de manière générale ${ }^{8}$. En plus de vouloir éduquer le regard de l'ouvrier et de l'étudiant pour que ceux-ci améliorent la qualité de leur production, le South Kensington Museum a aussi, sous la direction énergique de Henry Cole, tenté d'éduquer le goût du public, au moyen d'une muséographie ludique et claire. Le but de cette entreprise était de créer une exigence du côté des acheteurs, afin que l'artisan soit amené à se surpasser :

"I think the first result of this kind of exhibition is to make the public hunger after the objects; I think then they go to the china shops and say, "We do not like this or that; we have seen something prettier at the South Kensington Museum"; and the shop-keeper, who knows his own interest, repeat that to the manufacturer, and the manufacturer, instigated by that demand produces the article". "

Les vues de Henry Cole sont claires : le musée d'art industriel a pour but de servir le développement de l'industrie, et a de ce fait des visées commerciales pleinement assumées. Marius Vachon, lors de ses nombreuses enquêtes sur les

6. F. Mairesse, Le musée hybride, Paris, La Documentation Française, 2010, p. 50.

7. Sur I'histoire du South Kensington Museum voir Antony Burton, Vision and Accident, The story of the Victoria and Albert Museum, Londres, V\&A Publications, 1999.

8. Le musée présentait et publiait les œuvres de l'école, il était à l'initiative de concours nationaux et avait mis en place un système national de prêt d'œuvres, pour donner des modèles aux écoles de dessin provinciales. Voir à ce propos le rapport de Marius Vachon, Rapport sur les musées et les écoles d'art industriel en Angleterre, Missions de 1889 - Juin-Juillet, Paris, Imprimerie nationale, 1889.

9. «Je pense que le premier effet de ce type d'exposition est de rendre le public assoiffé d'objet ; je pense qu'ensuite ils se rendent chez les vendeurs de porcelaine [china étant le mot anglosaxon pour désigner la porcelaine] et dit "nous n'aimons pas ceci ou cela ; nous avons vu quelque chose de plus beau au South Kensington Museum", et le vendeur, dans son propre intérêt, le répète à l'artisan, et l'artisan, incité par cette demande, produit l'objet », Henry Cole, cité dans A. Burton, op. cit. note 7, p. 80. Traduction de l'auteur. 
musées industriels en $E_{u r o p e}{ }^{10}$, a également théorisé la nécessité pour le musée d'art industriel d'être avant tout un acteur commercial :

" Cette organisation nouvelle [les musées d'art et d'industrie], destinée à favoriser de la façon la plus pratique les intérêts des artistes et des chefs d'industrie, implique l'adoption d'un système de direction des musées entièrement nouveau qui va à l'encontre de nos idées actuelles sur ce sujet. Lérudition ne doit plus y jouer un rôle exclusif ; le directeur d'un musée d'art et d'industrie a le devoir de n'ignorer ni l'économie politique, ni la géographie, ni la science industrielle ; et s'il est très apte à mentionner sur un catalogue ou sur une pancarte les provenances, les dates et les attributions d'une faïence ancienne, il ne saurait désormais dédaigner d'inscrire au bas d'un étoffe moderne d'Orient ou d'Occident, d'une pièce de céramique, d'un bronze, etc., tous les renseignements commerciaux et industriels qui peuvent servir à un chef d'usine pour en connaître les procédés de fabrication, les conditions de vente et l'importance au point de vue de l'exportation ou du commerce intérieur ${ }^{11}$."

Aussi l'œuvre, au sein du musée d'art industriel, n'a pas le même statut que dans un musée des beaux-arts : elle se vêt d'une fonction à la fois éducative et commerciale, et devient en quelque sorte, un agent essentiel dans le développement des arts industriels de la nation.

\section{Les musées industriels en France dans le domaine de la céramique}

Les musées d'art industriel, bien qu'ayant pris le South Kensington Museum comme modèle commun ${ }^{12}$, ont chacun leurs spécificités, qu'il s'agisse de leurs dénominations ${ }^{13}$ ou de la nature de leurs collections. Tous les musées d'art industriel n'ont pas eu, comme lui, des collections encyclopédiques, certains s'étant spécialisés dans un domaine de production (céramique, textile, mécanique, etc.), choix bien évidemment guidé par la présence d'industries locales susceptibles d'être concernées. Dans le domaine de la céramique en France, deux musées furent créés sur les deux pôles de production de céramique de l'époque : Sèvres et Limoges. Le musée de Sèvres voit le jour dès 1824 à l'instigation d'Alexandre Brogniart (1770-1845), alors directeur de la manufacture impériale de porcelaine de Sèvres. Celui de Limoges est d'abord un musée départemental, aux collections hétéroclites rassemblées par la Société d'histoire et d'archéologie du Limousin ${ }^{14}$ et ne devient musée de la céramique qu'à partir de 1852, à l'initiative du préfet JeanBaptiste-Stanislas-Martial Migneret qui avait parfaitement saisi les enjeux que représentaient la création d'un tel musée dans une ville manufacturière telle que Limoges. Pris en charge par la mairie en 1867, le musée abandonne les quelques

10. Marius Vachon (1850-1928) réalise dans les années 1880-1890 une longue série d'enquêtes sur les musées et les écoles d'art industriel en Europe, dans le but de dresser un panorama de l'enseignement des arts industriels à son époque. Voir Stéphane Laurent, « Marius Vachon, un militant pour les "industries d'art" », dans Histoire de l'art, 29-30,1995, pp. 71-78.

11. M. Vachon, La crise Industrielle et artistique en France et en Europe, Paris, La Librairie Illustrée, 1886, pp. 126,127.

12. Sur l'influence du South Kensington Museum en Europe et dans le monde, voir le chapitre «South Kensington conquers the World » dans I'ouvrage de A. Burton, op. cit. note 7, pp. 104115 , ainsi que Michael Conforti, « The Idealist Enterprise and the Applied Arts », dans cat. d'exp., A grand design: the art of the Victoria and Albert Museum, 1997-1998, Baltimore Museum of Art; Boston, Museum of Fine Arts; Toronto, Royal Ontario Museum, New York, H. N. Abrams; Baltimore, The Baltimore Museum of Art, 1997, pp. 33-45.

13. «Musée d'art industriel » est un terme générique désignant des musées aux caractéristiques et aux objectifs communs. Mais les musées en question n'ont pas toujours répondu à cette dénomination : certains portent le nom de leurs lieux d'implantation (South Kensington Museum, dans le quartier de Kensington, Londres), d'autres le nom d'un membre bienfaiteur (Imperial Museum, Tokyo, musée Adrien Dubouché, Limoges), et lorsqu'il s'agit de nommer ces musées par la nature de leurs collections, apparaît alors une hésitation entre différents termes: le Museum für Kunst und Industrie de Vienne, I'Industrial Museum d'Edinburgh emploient le terme $\mathrm{d}^{\prime}$ « industrie », tandis que d'autres préfèrent à celui-ci le terme de «science » (Museum of Science and Art de Dublin), $d^{\prime} \ll$ arts appliqués », ou encore $d^{\prime} \ll$ arts décoratifs » (Musée des Arts Décoratifs de Paris).

14. Sur I'histoire du musée voir cat. d'expo., Adrien Dubouché, un musée un mécène, musée national Adrien Dubouché, 1990, sous la direction de Chantal Meslin-Perrier, Paris,

RMN, 1991, mais également Arnaud Denis, La genèse du Musée national Adrien Dubouché 18221881, (Mémoire de Muséologie de l'École du Louvre sous la direction de Dominique Poulot), 2001-2002. 
pièces exiguës de l'ancien Présidial pour un ancien asile d'aliénés, entièrement rénové à cette occasion (fig. 2).

Fig. 2

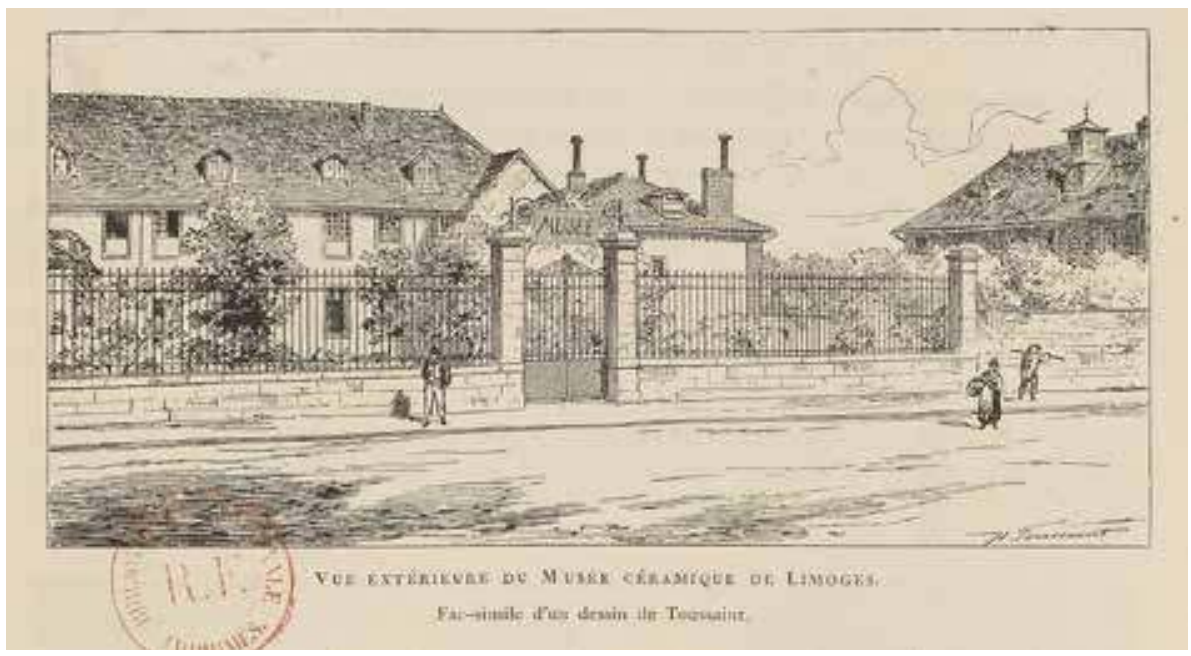

Une École des Beaux-arts est fondée en 1868, dans l'enceinte du musée. Les deux organismes sont sous la tutelle de l'emblématique Adrien Dubouché (1818$1881)^{15}$ à la fois directeur et donateur généreux.

\section{Les céramiques extrême-orientales du musée Adrien Dubouché de Limoges}

La collection extrême-orientale du musée Adrien Dubouché s'est essentiellement constituée au cours du XIX ${ }^{e}$ siècle, ce qui la rend très intéressante d'un point de vue historiographique : restée ainsi "dans son jus ", elle témoigne, aujourd'hui encore, non seulement du goût des acheteurs et des collectionneurs, mais également de l'avancée des connaissances en matière de céramique chinoise.

\section{Sortir de l'exotisme : les céramiques extrême-orientales comme modèle}

Les ouvres extrême-orientales, japonaises et chinoises, ont été jusqu'au $\mathrm{XVIII}^{\mathrm{e}}$ siècle des objets de collection et d'ornementation ${ }^{16}$. Dans un musée d'art industriel comme le musée de la céramique Adrien Dubouché, ces pièces prennent un sens nouveau, du fait des caractéristiques propres à ce musée, faisant de ces œuvres des modèles à étudier.

Les premières œuvres orientales du musée font leur apparition en 1850, cinq ans seulement après la fondation du musée. Il s'agit d'un don par Adrien Dubouché de vingt-cinq assiettes bleu et blanc datant des XVII ${ }^{\mathrm{e}}$ et $\mathrm{XVIII}^{\mathrm{e}}$ siècles, majoritairement chinoises, et réalisées pour l'exportation. Or, dès 1852, le musée change d'orientation grâce à l'impulsion du préfet Migneret, qui souhaite alors en faire, non pas un conservatoire d'objets anciens, mais bien un répertoire de modèles à même de former le goût du public comme celui des artistes. Le musée, contrairement aux « fabriques et [aux] magasins du commerce [...] où tout est dominé par les besoins du moment, [et où] l'on fabrique, l'on possède suivant les exigences du Commerce", permet la mise en place d'une "classification méthodique " offrant à l'œil de "saisir dans son ensemble le progrès absolu et

15. Né François-Louis Bourcin-Dubouché, Adrien Dubouché voit le jour dans une famille de riches négociants en draperie. En 1846, il épouse Ermance Bisquit et entre dans l'entreprise de cognac de son beau-père, qu'il fait prospérer. Son activité lui permet de consacrer sa fortune à l'achat d'œuvres pour le musée, ce qui lui vaudra d'être décoré Chevalier, puis Officier de la Légion d'honneur et Officier de l'instruction publique. Il fut également une personnalité éminente dans le monde de la céramique de l'époque, tantôt en tant que membre du Conseil supérieur de I'Union centrale, mais également en tant que membre du Conseil de perfectionnement de Sèvres. Voir C. Meslin-Perrier, op. cit. note 14, pp. 4-6 et 11 .

16. Voir supra note 2 . 
le progrès ou la décadence relatifs de la fabrication ${ }^{17}$ ". Les céramiques orientales furent ainsi intégrées à cette logique, et elles eurent dès cette époque le rôle de modèle au même titre que les céramiques européennes.

Le rôle de ces œuvres dans le musée est confirmé par l'action généreuse du directeur du musée Adrien Dubouché, qui a volontairement enrichi les collections extrême-orientales dans le but de donner aux artistes un panorama complet de la production céramique. En effet, il a été, au même titre que le préfet Migneret, très marqué par les idées de son temps à propos du rôle des musées dans le développement de l'industrie ${ }^{18}$, et par là même soucieux d'enrichir les collections de céramiques de manière à disposer, à Limoges, d'œuvres de toutes provenances et de toutes époques confondues. Dans cette optique, il a largement contribué à l'accroissement de la collection de céramiques extrême-orientales, en achetant lui-même des œuvres dont il a ensuite fait don au musée. Il considérait lui aussi les œuvres orientales comme des modèles à suivre et des sources d'inspiration, particulièrement en ce qui concerne les décors.

Les collections deviennent de véritables modèles à partir du moment où est créée en 1868, à l'initiative d'Adrien Dubouché, l'École des Beaux-arts de Limoges. Celle-ci gagne très vite en importance, jusqu'à devenir la raison d'être du musée, décrit dans certains textes comme une "annexe" de l'École ${ }^{19}$. De plus, l'École entretient des liens étroits avec les manufactures de porcelaine de Limoges, son financement étant dans les premiers temps assuré à part égale entre la ville de Limoges et les fabricants de porcelaine. Ces derniers prenaient également part à la gestion de l'École, puisqu'ils faisaient partie de son comité d'administration, composé également de membres du conseil municipal, de la Chambre de commerce, et de la Société des amis des arts ${ }^{20}$. Albert Guillemot ${ }^{21}$ résume bien les intentions d'Adrien Dubouché qui "voulait placer près de l'école un musée uniquement composé des plus belles pièces de porcelaine et de faïence qu'il serait possible de réunir ; elles devaient former non seulement pour les élèves, mais aussi pour les fabricants et les décorateurs de Limoges, comme une sorte de Conservatoire où tous les genres, tous les types seraient rassemblés, depuis les amphores du musée Campana jusqu'aux productions les plus récentes de la Chine et du Japon ${ }^{22}$.»

Un événement crucial vient confirmer le rôle fondamental des céramiques extrême-orientales comme modèle. Il s'agit de la participation de l'École des Beaux-arts de Limoges à l'exposition du Musée Oriental ${ }^{23}$ de l'Union Centrale des Arts appliqués à l'Industrie (UCAD) de 1869, associant création contemporaine et œuvres anciennes chinoises et japonaises issues de collections privées. Dans une lettre adressée au jury, Adrien Dubouché précise en quoi les œuvres peuvent être prises comme modèle, c'est selon lui le décor qui mérite le plus d'attention :

" ...pour les ornements et le décor, nous tâchons de combiner la tradition classique avec la tradition orientale si libre, si élégante, si remplie d'imagination, inépuisable en fantaisie et en caprices. Il est indispensable à de futurs décorateurs de connaitre et d'étudier à fond ces arts de l'Orient

17. Voir la lettre du préfet Migneret au maire de Limoges en date du 7 octobre 1852 sur la création d'une section céramique au sein du musée départemental, Archives Municipales de Limoges - Boîte 2 R 21.

18. Voir Adrien Dubouché, «Le musée céramique de Limoges », Gazette des Beaux-arts, $16^{\mathrm{e}}$ année, T. X, $2^{\mathrm{e}}$ période, pp. 225-237, et du même auteur : Notice sur les écoles gratuites des beaux-arts appliqués à l'industrie, Paris, J. Claye, 1869. Voir également les écrits de Henry Cole. 19. Il est désigné comme tel dans le projet de loi ayant pour objet la création d'une école nationale d'art décoratif et d'un musée national de Limoges publié dans le Journal Officiel du 22 février 1881.

20. Albert Guillemot, «Adrien Dubouché », Revue des Arts décoratifs, 1881-1882, tome 2, p. 214.

21. Rédacteur en chef du Courrier du centre, membre de la Société Archéologique et Historique du Limousin, et membre de la direction du Musée de Céramique (futur musée Adrien Dubouché), l'auteur souhaite remercier ici Valérie Gramfort pour sa précieuse aide documentaire.

22. A. Guillemot, op. cit. note 20, p. 215 .

23. Appellation qui n'est pas sans rappeler celle employée au musée South Kensington de Londres, dont les différents départements étaient divisés en autant de museum : Museum of Education, Museum of Art, Museum of Architecture, etc. 
dont l'Union Centrale a précisément réuni cette année de si merveilleux

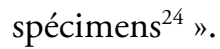

Ainsi, les aspirations du musée, sa politique d'acquisition, et le lien étroit qu'il entretient avec l'École des Beaux-arts, contribuent pleinement à la redéfinition du statut de l'œuvre dans le musée, ce qui dans le cadre des céramiques extrêmeorientales présente une nouveauté par rapport à leur antécédent d'objet exotique voué à l'ornementation.

\section{Des œuvres comme support de la connaissance:}

le musée de Limoges et le cercle des collectionneurs parisiens

Sans doute conscient de ses lacunes dans le domaine, et de son manque de disponibilité, Adrien Dubouché a très tôt confié à des collectionneurs parisiens plus avisés en matière de céramiques chinoises et japonaises le soin de choisir et d'acquérir des œuvres pour le musée, et ce grâce à ses ressources financières personnelles. En retraçant l'arrivée de ces collections dans le musée, il est intéressant de remarquer que ces acquisitions correspondent, d'une certaine manière, à l'avancée des connaissances dans le domaine de la céramique chinoise. Adrien Dubouché était très proche du petit cercle de céramologues parisiens, et c'est auprès des collectionneurs les plus fameux qu'il parvient à obtenir des œuvres pour le musée. Les deux personnalités les plus influentes dans le monde de la collection sont Albert Jacquemart (fig. 3) et Paul Gasnault, tous deux ayant contribué à la constitution d'un savoir sur les céramiques chinoises et japonaises grâce à leurs nombreuses publications. L'entrée de ces collections dans le musée est significatif de l'avènement de ce savoir, soit parce qu'elles représentent un nouveau type d'œuvres jusqu'alors peu présent dans les collections, soit parce qu'elles seront exposées dans les salles chronologiquement, le classement en permettant la compréhension. La muséalisation entraîne là encore une transformation du statut de l'œuvre dans le musée qui est alors considérée comme un objet d'étude.

Fig. 3 d'Albert Jacquemart Gravure à I'eau forte d'après Jacquemart A. Catalogue de la collection Jacquemart Paris, Typographie Charles Usinger, 1879,

Paris, Bibliothèque nationale de France

(c)Bibliothèque nationale de France
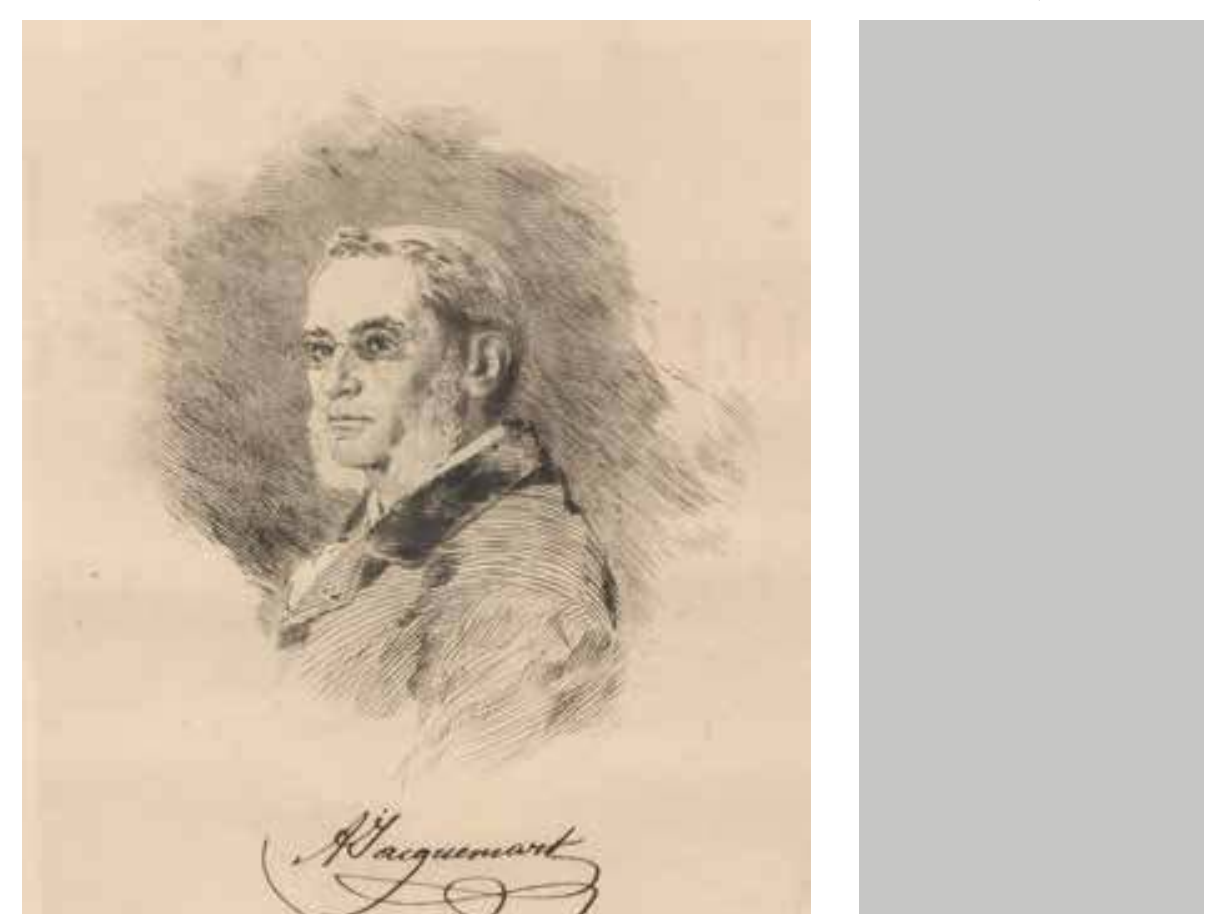

À partir de 1869, Adrien Dubouché mandate Jules Michelin, artiste peintre afin que celui-ci acquière des œuvres à Paris ${ }^{25}$. Sans doute grâce à ses conseils, de

24. Adrien Dubouché, Limoges, École gratuite des Beaux-arts appliqués à l'Industrie, Paris, J. Claye, 1869, p. 14.

25. Voir Laure Chabanne, « La porcelaine chinoise dans les collections du musée national Adrien Dubouché, à Limoges », dans L'Odyssée de la porcelaine chinoise, (cat. d'expo., Musée national de la céramique, Sèvres ; Musée national Adrien Dubouché, Limoges et Musée de la Faïence Château Pastré, Marseille, 2003-2004), Paris : Réunion des Musées nationaux, 2003, pp. 9-14. 
nouveaux types d'œuvres font leur apparition dans le musée : les monochromes chinois, des œuvres plus anciennes (seconde moitié du XviI ${ }^{\mathrm{e}}$ siècle) dans le style rouge et vert, des blanc de chine (fours de Dehua - 德化), ainsi qu'un ensemble de théières de Yixing (宜興). Des pièces seront aussi acquises auprès de Paul Gasnault.

Adrien Dubouché acquiert l'importante collection de son ami Albert Jacquemart ${ }^{26}$ à la mort de celui-ci en 1875 , avant même qu'il soit question de la mettre en vente ${ }^{27}$. La valeur de cette collection réside moins dans son nombre, que dans la manière dont elle fut constituée, et dans sa cohérence en tant qu'ensemble. Albert Jacquemart était en effet un collectionneur scientifique, et minutieux, un "inventeur " comme se plaît à l'appeler son disciple et ami Paul Gasnault ${ }^{28}$. Il est à l'origine, avec Edmond Le Blant, de la toute première tentative de classification de la porcelaine chinoise, en langue française ${ }^{29}$. Dans cet ouvrage, les deux auteurs mettent au point un système de compréhension des céramiques chinoises et japonaises en les rassemblant, soit en fonction de leurs inscriptions pour les pièces inscrites, soit selon leurs décors (émaux employés ou motifs). Plus tard, il continuera de publier seul sur la céramique ${ }^{30}$, se servant de sa collection (mais également de celles de ses amis comme Paul Gasnault) pour illustrer les planches de ses ouvrages (fig. 4), et montrer son classement. Sa collection est le reflet de son système, elle en est la véritable démonstration.

Albert Jacquemart n'a pas seulement pensé son classement au moyen de sa collection, il a également, à l'inverse, complété sa collection selon ce classement. Il s'est attaché à obtenir des œuvres représentatives d'un type, plutôt que des chefs-d'œuvre. Par exemple, il a souhaité acquérir au moins une pièce inscrite pour chaque nom d'empereur de façon à reconstituer une chronologie parfaite des dynasties Ming (明代, 1368-1644) et Qing (清代, 1644-1911). Paul Gasnault en témoigne dans sa préface au catalogue de la collection Jacquemart :

" Il est bien certain qu'il y a dans cette collection plus d'une pièce mutilée, peu séduisante pour l'œil du visiteur superficiel, et dont pourtant la découverte a causé à Albert Jacquemart plus de joie triomphante que n'en avait jamais éprouvé amateur à la vue de l'objet le plus splendide et le plus merveilleusement conservé31".

Aujourd'hui encore certains termes tels que "famille verte» ou "famille rose", instaurés par Jacquemart, sont restés en usage en français comme en anglais. Son classement a en revanche été très vite critiqué par d'autres céramologues peu de temps après sa parution ${ }^{32}$, en

26. Albert Jacquemart (1808-1875). Il se forme au dessin dans l'atelier de $M$. Buffet puis à I'École des Beaux-Arts. Il réalise les planches de l'anatomiste Bazin, puis des planches pour la bibliothèque du Museum du Jardin des Plantes. Jacquemart s'adonne un temps à la botanique et publie dans ce domaine deux volumes: La Flore des Dames en 1840, et le Nouveau Langage des Fleurs en 1841. En 1825, il rentre dans l'administration des douanes, pour des raisons sans doute financières, où il devient chef de bureau. C'est là qu'il fait la rencontre d'Edmond Le Blant, camarade d'administration avec lequel il partage la même passion pour les céramiques chinoises ; voir Henry Perrier, « Une vie bien remplie », L'art, 1876, T.4, pp. 10-13. (Albert Jacquemart et sa collection font actuellement l'objet de la thèse doctorale de l'auteur).

27. C'est à partir de ce don que le musée prend le nom de son directeur, comme gage de reconnaissance de la ville de Limoges.

28. Paul Gasnault, « La collection Jacquemart et le musée de la céramique de Limoges », L'Art, 1876, p. 4.

29. Albert Jacquemart, Edmond Le Blant, Histoire artistique, industrielle et commerciale de la Porcelaine : accompagnée de recherches sur les sujets et emblèmes qui la décorent, les marques \& inscriptions qui font reconnaître les fabriques d'où elle sort, les variations de prix qu'ont obtenu les principaux objets connus \& les collections où ils sont conservés aujourd'hui, 2 vol., Paris, J. Techener, 1861-1862.

30. A. Jacquemart, Les merveilles de la céramique : ou l'art de façonner et décorer les vases en terre cuite, faïence, grès et porcelaine, depuis les temps antiques jusqu'à nos jours, 3 vol., Paris, Hachette, 1866-1869, et du même auteur, Histoire de la céramique : étude descriptive et raisonnée des poteries de tous temps et de tous les peuples, Paris, Librairie Hachette et Cie, 1873, 750 p., XII pl.

31. P. Gasnault, Catalogue de la collection Jacquemart, Paris, Librairie de I'art, A. Ballue éditeur, 1879 , p. IX.

32. Édouard Garnier remet en question le classement de Jacquemart dans son Histoire de la Céramique, Poteries, Faïences et Porcelaines chez tous les peuples depuis les temps anciens jusqu'à nos jours, Tours, Alfred Mame et fils, 1882, p. 403. En Angleterre, c'est le futur directeur du British Museum, Augustus Wollaston Franks, qui souligne les erreurs d'attribution de ce classement dans son rapport sur la céramique japonaise en 1880, voir Anna Somers Cocks, The Victoria and Albert Museum. The making of the collection, Leicester, Windward, 1980, p. 132. 
raison des erreurs d'attribution et des approximations qu'il comportait.

Fig. 4

Jules Jacquemart, Planche VIII Famille rose japonaise «École artistique » Gravures à l'eau forte 1861 ,

d'après I'Histoire artistique, industrielle et commerciale de la Porcelaine de Le Blant et Jacquemart (les objets représentés ci-dessus correspondent aux œuvres du musée Adrien Dubouché ADL 1865 soucoupe à décors de coq AD 1827 petite tasse à décors abstraits) Bibliothèque nationale de France Bibliothèque nationale de France (c)Bibliothèque nationale de France Toussaint
Musée Adrien Dubouché « Vue de l'intérieur du Musée céramique de Limoges », ancien Asile des aliénés

Gravure à l'eau forte Fac-similé d'après la revue $L$ 'Art 1876, t. 6 , p. 170 Paris, Bibliothèque nationale de France
FOL-V-29 (c)Bibliothèque nationale de France
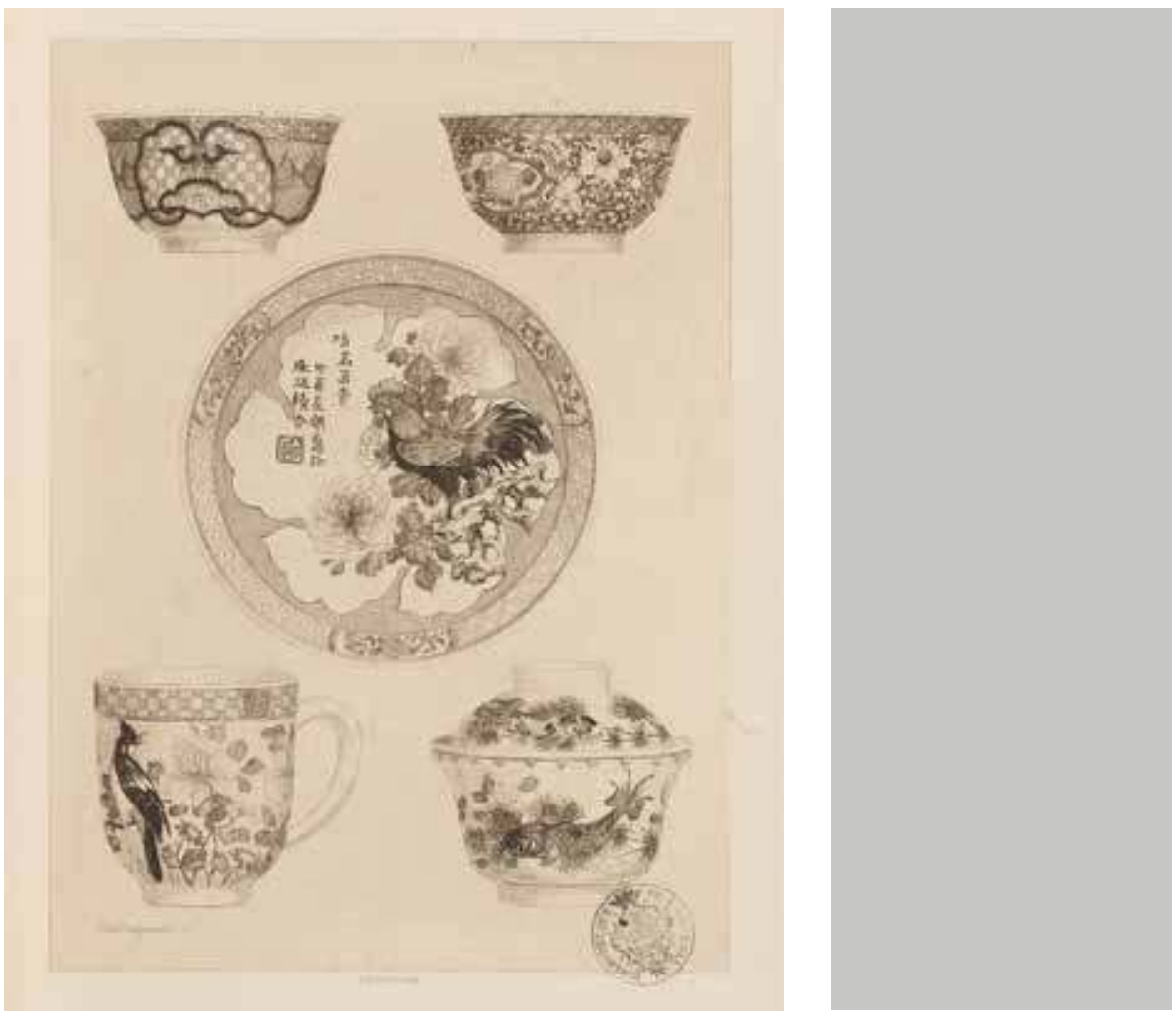

Les clauses exigées par les héritiers de Jacquemart au moment de la vente, précisaient que la collection devait être présentée dans son intégralité, selon le classement initié par celui-ci ${ }^{33}$. À Limoges, un espace est spécialement aménagé pour recevoir la collection, grâce à l'allocation d'une subvention de la mairie ${ }^{34}$. La dernière salle du musée (fig. 5), qui était jusqu'alors occupée par des peintures, est ainsi entièrement dédiée à la collection Jacquemart, et portera désormais son nom.

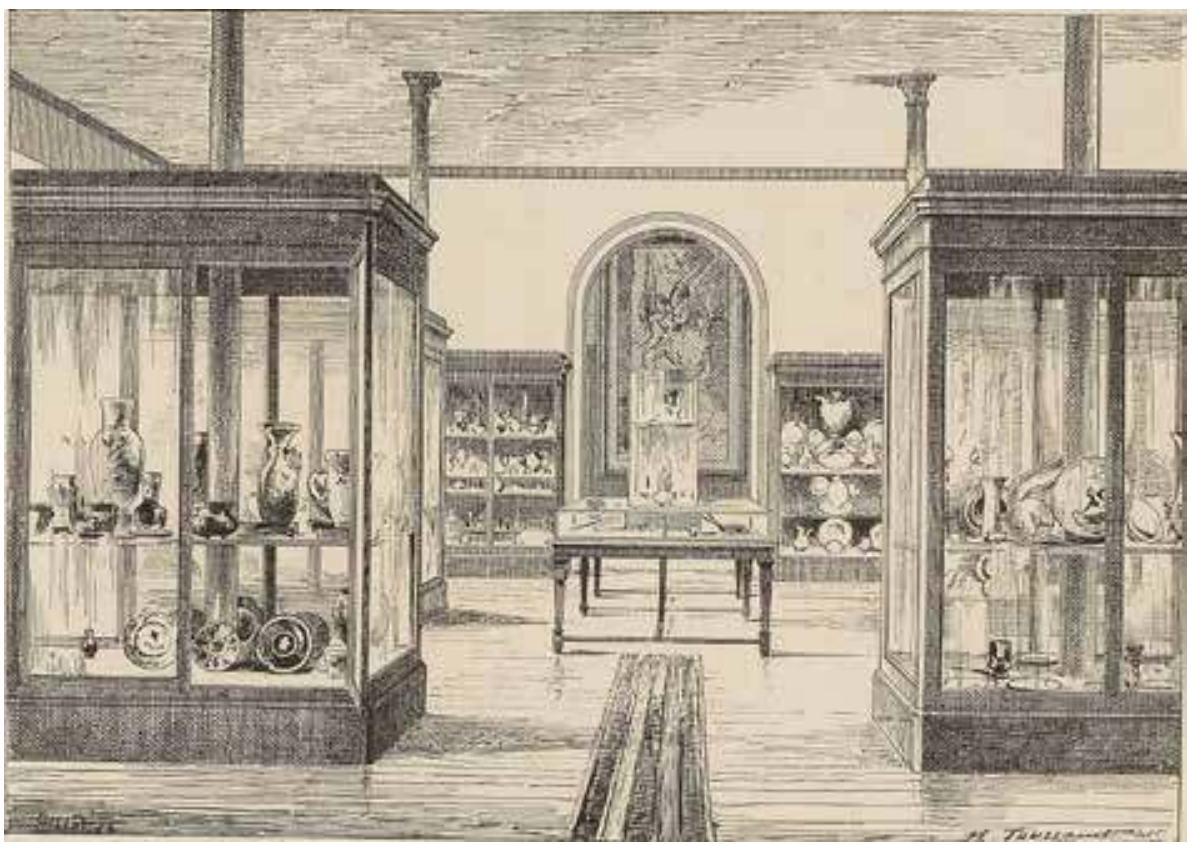

33. Voir lettre d'Adrien Dubouché au maire de Limoges en date du 30 déc. 1875, Archives municipales de Limoges, série RI (271) stipulant que : « les clauses du contrat de cette vente sont que la salle qui recevra cette collection portera le nom d'Albert Jacquemart et que chaque objet sera placé comme l'avait fait son créateur, avec la méthode et les divisions qu'il a indiquées lui-même ».

34. Extrait du registre des délibérations du conseil municipal de la commune de Limoges, séance du 11 nov. 1876, Archives municipales de Limoges, série RI (271). 
Si l'on en croit un guide publié dans les années 1880 , les collections de céramiques extrême-orientales étaient toutes rassemblées dans la dernière vitrine du musée $\left(n^{\circ} 52\right)$.

Sous l'impulsion d'Adrien Dubouché, la collection Jacquemart entre au musée de Limoges comme un ensemble complet. Ce processus de muséalisation, n'implique pas seulement les œuvres en elles-mêmes, mais également le classement de Jacquemart, auquel la présentation rend honneur. Ainsi le musée accompagne, voire même valide un savoir, un système de compréhension qu'il rend accessible à tous, en tant qu'institution publique faisant autorité dans le domaine de l'histoire de l'art.

À peine quelques années plus tard, Adrien Dubouché fait une fois de plus preuve d'une grande générosité envers le musée, en offrant la collection de Paul Gasnault, après l'avoir achetée à ce dernier. C'est la deuxième grande collection d'art extrême-oriental du musée. Paul Gasnault l'a constituée dans la même optique que son aîné Albert Jacquemart, acquérant parfois des œuvres qui ne valaient que pour le procédé technique qu'elles permettaient de démontrer, mais il n'a pas conservé le classement original de Jacquemart, conscient des failles qu'il présentait. Devenant conservateur du musée Adrien Dubouché après la mort de ce dernier en 1881, il prend pleinement part à l'organisation de la collection dans les salles du musée. Paul Gasnault n’a pas souhaité que sa collection soit présentée comme un tout, préférant l'intégrer aux collections du musée.

On note quelques éléments intéressants du point de vue de la muséographie, notamment le fait que certaines collections asiatiques soient présentées dans les mêmes vitrines que les collections contemporaines des manufactures de Limoges ${ }^{35}$. Il semble évident qu'un tel rassemblement était fait pour montrer une filiation entre la production limougeaude ${ }^{36}$ et la céramique chinoise.

Aussi, à la fin du siècle, il semble bien que l'exposition de modèles chinois ait porté ses fruits et que les manufactures en aient intégré les techniques. L'objet qu'est la céramique chinoise, s'est doté de plusieurs statuts en passant entre les mains des collectionneurs puis celles des conservateurs. Il est important de constater qu'à partir du XIX ${ }^{e}$ siècle, ces œuvres ont perdu leur fonction d'ornement exotique pour devenir des objets d'étude, qu'il s'agisse de modèle pour inspirer la création contemporaine, ou bien de support de la connaissance au moment où s'écrivent les premiers ouvrages sur l'histoire de la céramique chinoise. Le musée joue un rôle fondamental dans ce processus : il acquiert, valide cette connaissance, la rend visible et appréciable par tous. La collection d'art asiatique du musée de la Céramique de Limoges telle qu'elle s'est constituée retrace ainsi les premiers pas de la constitution d'un savoir. La transformation du statut de l'objet opère un dernier tournant au début $\mathrm{du} \mathrm{xx}^{\mathrm{e}}$ siècle, alors que les musées d'art industriel perdent peu à peu leur vocation initiale pour devenir des musées d'arts décoratifs. Le glissement qui s'effectue entre le musée d'art industriel, si essentiel au progrès de l'industrie et de la nation des années 1850-1880, et le musée des arts décoratifs, entraîne consécutivement une remise en question de la place des œuvres dans le musée. Celles-ci perdent leur valeur de modèle d'inspiration pour devenir, au même titre que les œuvres des musées de beaux-arts, des œuvres d'art. Grâce à leur exposition dans le musée d'art industriel, les œuvres chinoises sont passées du statut d'objet de curiosité à celui de modèle à imiter, pour enfin être reconnues en tant qu'œuvres d'art. Au cours de ce processus s'est également tissée une histoire de l'art de la céramique chinoise, qui malgré son caractère empirique, ne constitue rien moins que les premiers jalons d'une meilleure compréhension de l'art chinois.

35. La muséographie de cette époque peut être reconstituée dans ses grandes lignes principalement grâce à deux documents, le premier est l'inventaire dressé par Paul Gasnault en 1881 conservé aux Archives municipales de Limoges (série RI [271]), qui permet de connaître le contenu des vitrines, mais également un article de Camille Lemaire, « Une promenade au Musée national Adrien Dubouché », Almanach Limousin, 1884, p. 110-123.

36. Ce rapprochement était dans la vitrine 4 , et concernait la manufacture Haviland (Limoges et Auteuil). 


\section{L'auteur}

Pauline d'Abrigeon est diplômée de l'École du Louvre et de l'Institut national de langues et civilisations orientales (Inalco) section chinoise. Elle a réalisé son Master de recherche en muséologie sous la direction de François Mairesse, interrogeant le statut des œuvres extrême- orientales dans les musées industriels. Aujourd'hui, elle poursuit ses recherches associant muséologie et histoire de l'art chinois à la National Taiwan University (NTU). 